\title{
Evaluation of an Institutional Project to Improve Venous Thromboembolism Prevention
}

\author{
Christina A. Minami, MD,2, Anthony D. Yang, MD¹, Mila Ju, MD, MS¹, Eckford Culver, BS³, Kathryn Seifert, BS³, \\ Lindsey Kreutzer, MPH${ }^{1}$, Terri Halverson, BS ${ }^{3}$, Kevin J. O’Leary, MD, MS ${ }^{4}$, Karl Y. Bilimoria, MD, MS ${ }^{1,2 *}$
}

\begin{abstract}
'Surgical Outcomes and Quality Improvement Center, Department of Surgery, Feinberg School of Medicine, Northwestern University and Northwestern Memorial Hospital, Chicago, Illinois; '2Center for Healthcare Studies, Feinberg School of Medicine, Northwestern University, Chicago, Illinois; ${ }^{3}$ Northwestern Memorial Hospital, Chicago, Illinois; ' ${ }^{4}$ ivision of Hospital Medicine, Feinberg School of Medicine, Northwestern University, Chicago, Illinois.
\end{abstract}

BACKGROUND: Northwestern Memorial Hospital (NMH) was historically a poor performer on the venous thromboembolism (VTE) outcome measure. As this measure has been shown to be flawed by surveillance bias, $\mathrm{NMH}$ embraced process-of-care measures to ensure appropriate VTE prophylaxis to assess healthcare-associated VTE prevention efforts.

OBJECTIVE: To evaluate the impact of an institution-wide project aimed at improving hospital performance on VTE prophylaxis measures.

DESIGN: A retrospective observational study.

SETTING: NMH, an 885-bed academic medical center in Chicago, Illinois

PATIENTS: Inpatients admitted to NMH from January 1, 2013 to May 1, 2013 and from October 1, 2014 to April 1, 2015 were eligible for evaluation.

INTERVENTION: Using the define-measure-analyzeimprove-control (DMAIC) process-improvement methodology, a multidisciplinary team implemented and iteratively improved 15 data-driven interventions in 4 broad areas: (1) electronic medical record (EMR) alerts, (2) education initiatives, (3) new EMR order sets, and (4) other EMR changes.
MEASUREMENTS: The Joint Commission's 6 core measures and the Surgical Care Improvement Project (SCIP) SCIP-VTE-2 measure.

RESULTS: Based on 3103 observations (1679 from January 1, 2013 to May 1, 2013, and 1424 from October 1, 2014 to April 1, 2015), performance on the core measures improved. Performance on measure 1 (chemoprophylaxis) improved from $82.5 \%$ to $90.2 \%$ on medicine services, and from $94.4 \%$ to $97.6 \%$ on surgical services. The largest improvements were seen in measure 4 (platelet monitoring), with a performance increase from $76.7 \%$ adherence to $100 \%$, and measure 5 (warfarin discharge instructions), with a performance increase from $27.4 \%$ to $88.8 \%$.

CONCLUSION: A systematic hospital-wide DMAIC project improved VTE prophylaxis measure performance. Sustained performance has been observed, and novel control mechanisms for continued performance surveillance have been embedded in the hospital system. Journal of Hospital Medicine 2016;11:S29-S37. (c) 2016 Society of Hospital Medicine
Venous thromboembolism (VTE), which includes deep vein thrombosis (DVT) and pulmonary embolism, is a significant cause of morbidity and mortality in the United States among hospitalized patients. ${ }^{1-6}$ Although it may not be possible to completely eradicate VTE events, ${ }^{7}$ chemical and/or mechanical prophylaxis can reduce VTE rates by up to $74 \%$ to $86 \%,{ }^{8-10}$ and meta-analyses have demonstrated the benefit of VTE prophylaxis in the inpatient population. ${ }^{11,12}$ Despite evidence-based guidelines regarding the appropriate type, duration, and dosing of

\footnotetext{
*Address for correspondence and reprint requests: Karl Y. Bilimoria, MD, Surgical Outcomes and Quality Improvement Center, Department of Surgery, Feinberg School of Medicine, Northwestern University, 633 N St. Clair, 20th Floor, Chicago, IL 60611; Telephone: 312-695-4853;

Fax: 312-503-4401; E-mail: k-bilimoria@northwestern.edu

Additional Supporting Information may be found in the online version of this article.

Received: June 16, 2016; Revised: August 25, 2016; Accepted: August 28,2016

2016 Society of Hospital Medicine DOI 10.1002/jhm.2663

Published online in Wiley Online Library (Wileyonlinelibrary.com).
}

prophylaxis, thromboprophylaxis has been found to be underutilized in the inpatient setting. ${ }^{13-15}$

Northwestern Memorial Hospital (NMH) historically performed poorly on VTE outcome measures. VTE in the surgical patient population was an especially glaring problem, as NMH was persistently found to be a risk-adjusted poor performer in the American College of Surgeons National Surgical Quality Improvement Project (ACS-NSQIP).

However, VTE outcome measures have been shown to be problematic due to their susceptibility to surveillance bias; that is, variation in the ordering of screening or diagnostic VTE imaging studies between hospitals leads to variable VTE rates (the more you look, the more you find). ${ }^{16-19}$ More vigilant hospitals that have a lower threshold to order an imaging study may find higher occurrences of VTE, and paradoxically be deemed a poor performer. Surveillance bias and the lack of validity of the VTE outcome measurement highlighted the importance of utilizing process-of-care measures in assessing hospital VTE prevention efforts. $^{20,21}$ Thus, when the Joint Commission enacted 
6 new VTE core process-of-care measures on January 1, 2013 to monitor hospital performance on VTE prophylaxis administration and VTE treatment (Table 1), $\mathrm{NMH}$ undertook a hospital-wide quality-improvement (QI) project utilizing the define-measure-analyzeimprove-control (DMAIC) process improvement (PI) methodology to optimize their performance on these core measures as well as the Surgical Care Improvement Project (SCIP) SCIP-VTE-2 measure. In this article, we describe the QI effort undertaken at $\mathrm{NMH}$ to improve hospital-level measure performance and the outcomes of this effort.

\section{METHODS}

\section{Setting}

$\mathrm{NMH}$ is a tertiary referral and teaching hospital affiliated with the Feinberg School of Medicine of Northwestern University. It is the flagship of Northwestern Medicine, which also includes 4 community hospitals, a dedicated women's hospital, and outpatient and urgent care centers. ${ }^{22} \mathrm{NMH}$ is an 885 -bed hospital with approximately 50,000 inpatients admitted annually. This project, to evaluate the outcomes of the NMH VTE QI initiative, was reviewed and approved by the Northwestern University Institutional Review Board as an exempt activity.

\section{Measures}

The Joint Commission VTE measures were a product of the National Consensus Standards for the Prevention and Care of Deep Vein Thrombosis project between the Joint Commission and National Quality Forum (NQF). These 6 measures are endorsed by the NQF and aligned with the Centers of Medicare and Medicaid Services. ${ }^{23}$ SCIP also has measures focusing on VTE prophylaxis. SCIP-VTE-2 focuses on prophylaxis in the perioperative period (the 24 hours prior to anesthesia start time to 24 hours postanesthesia end time). Specific measure definitions are in Table 1. All patients hospitalized at NMH were eligible for case abstraction; specific inclusion and exclusion criteria were based on measure specifics set forth by The Joint Commission and SCIP, and random cases were selected for abstraction utilizing the standard sampling methodology required for these measures. Case abstraction was performed by a nurse and validated by physicians.

\section{The Intervention}

Review of baseline performance on the core measures began in January 2013. Common failure points were identified first by electronic medical record (EMR) evaluation. Subsequently, focus groups with front-line staff, close examination of EMR ordering logic for chemical and mechanical prophylaxis with the IT department, hospital floor observations, and evaluation of the patient education process during discharge were performed to further define the reasons for common failure points.

Fifteen data-driven, focused interventions were then designed, pilot tested, and implemented throughout the hospital in May 2013, with iterative improvement of each component over the next 18 months (Table 1). This project utilized DMAIC PI methodology, and was carried out by a multidisciplinary team with representatives from the departments of surgery, internal medicine, anesthesia, gynecology, PI, clinical quality, pharmacy, analytics, information technology (IT), and nursing. Broadly, the 15 interventions consisted of (1) EMR alerts, (2) education initiatives, (3) new EMR order sets, and (4) other EMR changes.

\section{EMR Alerts}

Novel provider alerts were built into NMH's inpatient EMR platform (Cerner PowerChart; Cerner Corp., North Kansas City, MO) to address common mistakes contributing to failures on VTE-1 (chemoprophylaxis) and VTE-3 (overlap therapy). Although VTE-1 failures were often multifactorial, missing documentation regarding reasons for no chemoprophylaxis given and failures to order chemoprophylaxis were 2 common drivers of failures. To address these 2 problems, a logic-driven alert to force patient-specific ordering of appropriate VTE prophylaxis was developed (Figure 1). VTE-3 (overlap therapy) failures occurred due to clinician failure to order a full 5 days of overlap therapy when switching from parenteral anticoagulation to warfarin therapy; hence, to target VTE-3 performance, new alerts reminding clinicians to meticulously order and document the overlap of parenteral VTE therapy and warfarin were developed. As part of the logicdriven alert to improve patient-specific ordering of appropriate VTE prophylaxis, we allowed for the inclusion of documentation of a contraindication to explain why VTE prophylaxis was not ordered.

\section{Educational Initiatives}

After consulting with attending physicians, residents, nurses, and practice managers at NMH to understand the potential drivers of VTE-1 (chemoprophylaxis) failures, a team of clinicians and PI experts held 2part interactive educational sessions with nurses to address knowledge deficits. The first part focused on general VTE education (eg, the significance of the problem nationwide as well as at $\mathrm{NMH}$, general signs and symptoms of VTE, risk factors for VTE, and $\mathrm{NMH}$-specific failure rates for mechanical and chemoprophylaxis). The second portion used a myth-busting approach, in which common misunderstandings that frequently impede VTE prophylaxis (eg, a patient capable of ambulating does not need sequential compression devices (SCDs), or SCDs cannot be applied to a patient with acute or chronic DVT) were discussed. Educational efforts also addressed VTE-5 (warfarin discharge instructions) performance; 
Improving VTE Prevention | Minami et al

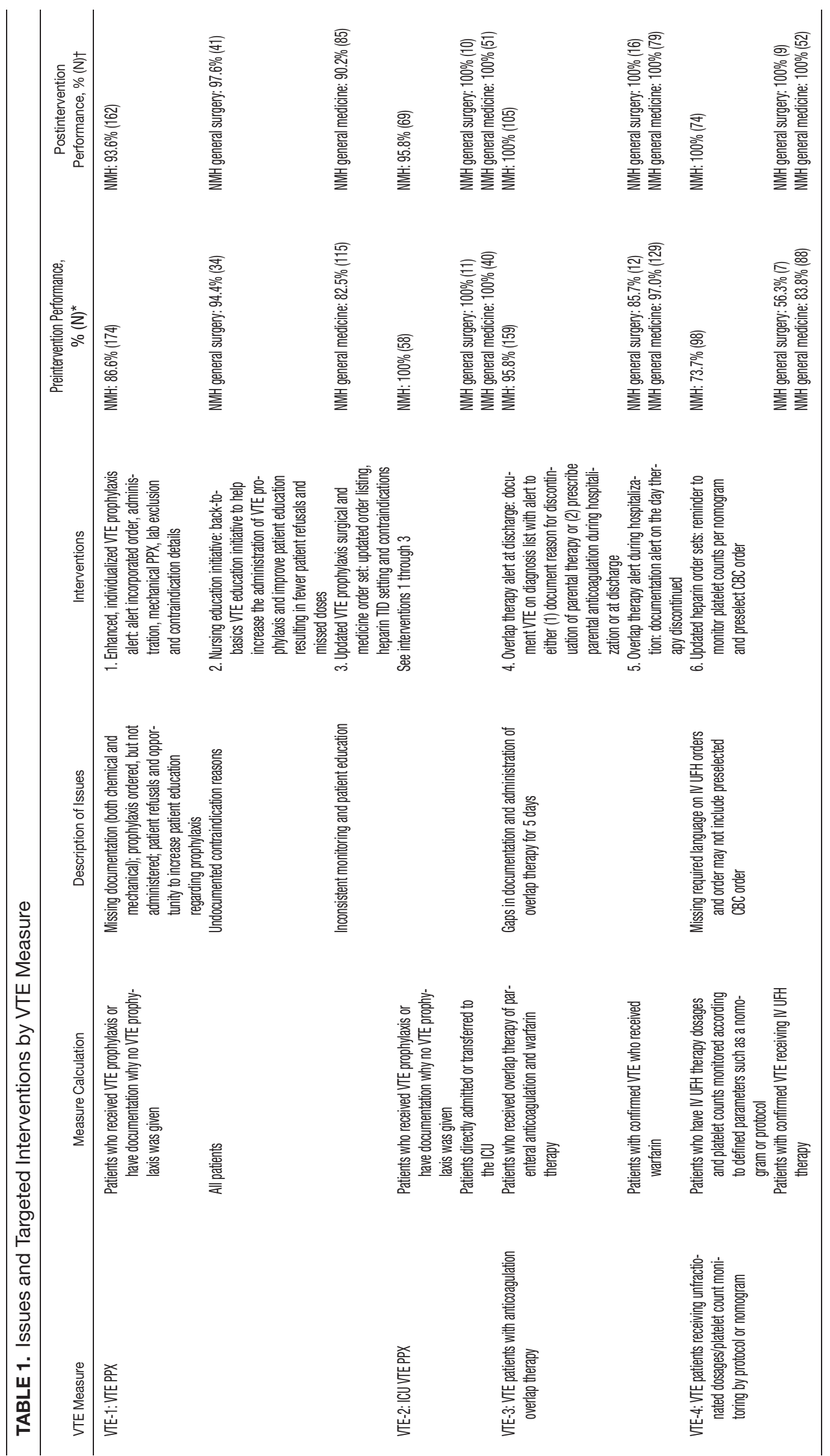




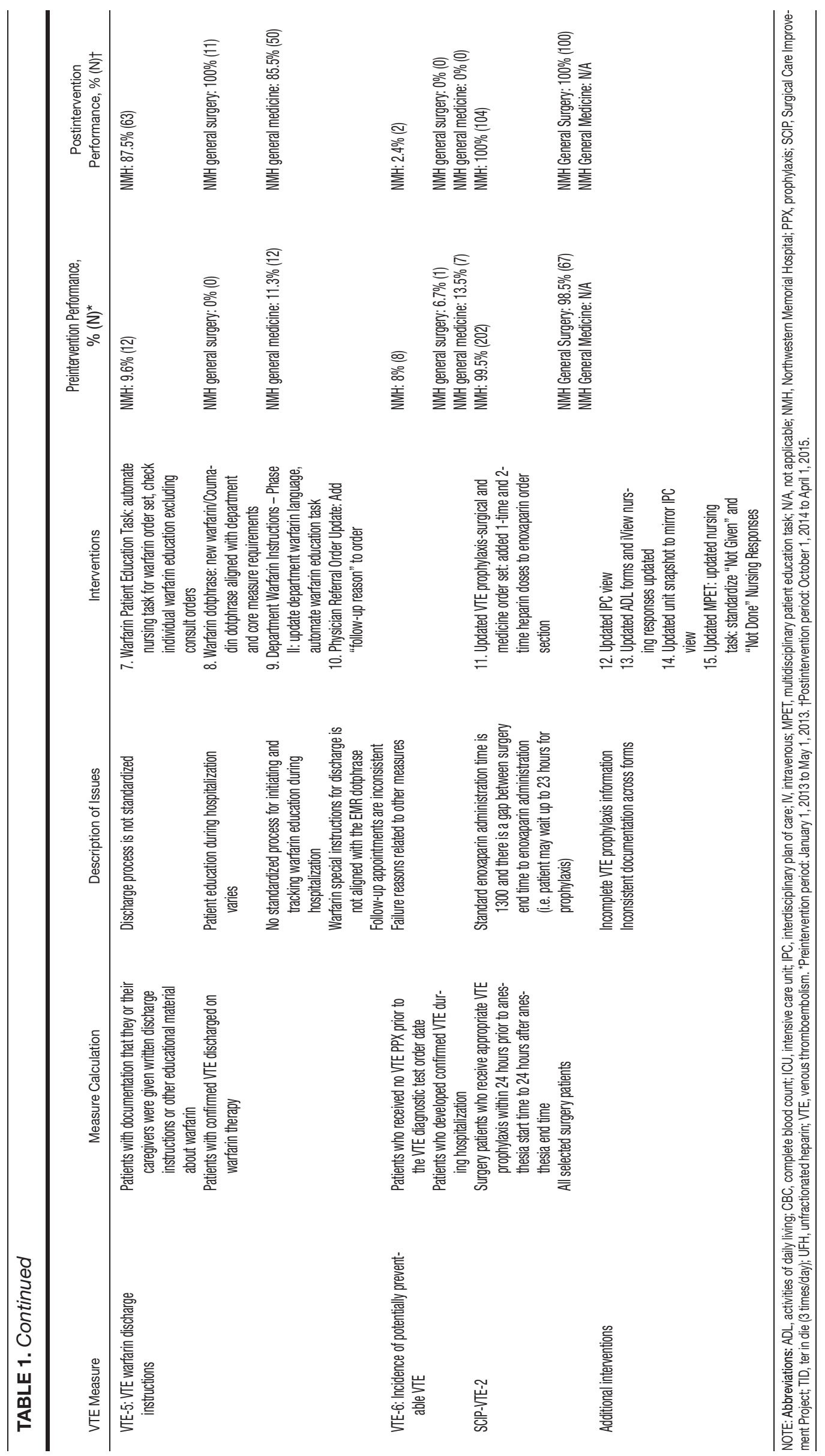




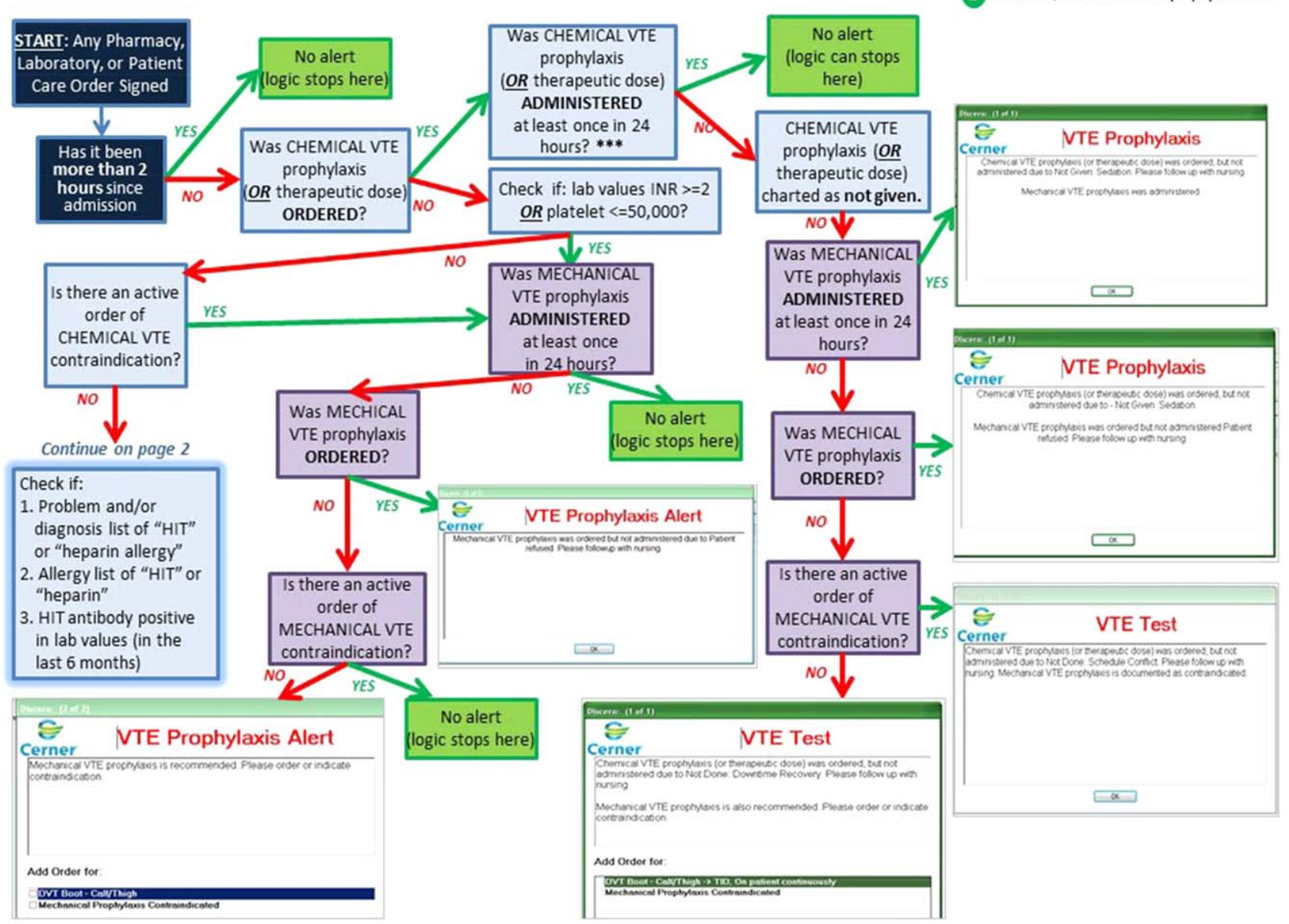

FIG. 1. Map of EMR logic for VTE prophylaxis alerts. Abbreviations: EMR, electronic medical record; HIT, heparin-induced thrombocytopenia; INR, international normalized ratio; VTE, venous thromboembolism.

although nurses provided patient education with regard to home warfarin use, the timing was inconsistent. The VTE-5 education provided nurses with a standardized method and time for educating patients about postdischarge warfarin use. EMR changes ensured that when warfarin was ordered, warfarin education automatically populated the nurse's task list, reminding them to educate their patients prior to discharge.

\section{New EMR Order Sets}

Previously existing order sets often made it difficult for physicians to order the correct dosing and timing of VTE prophylaxis, document contraindications to prophylaxis, and lacked the appropriate laboratory orders with therapy orders. New order sets were designed to facilitate compliance with VTE-1 (chemoprophylaxis), VTE-4 (platelet monitoring), VTE-5 (warfarin discharge instructions), and SCIP-VTE-2 (perioperative prophylaxis) by updating lab and medication order listings, dosing choices, prophylaxis contraindications, reminders to monitor platelet counts per nomogram, and physician follow-up reasons. When we considered our hospital's specific local factors, we came to the conclusion that risk stratification would be a difficult strategy to apply effectively as a component of the new order sets, mainly due to barriers related to buy-in from physicians and nurses.

\section{Other EMR Changes}

Other interventions targeted at specific issues were programmed into the EMR. For example, a shortcut (known as a dotphrase in Cerner PowerChart) for inserting warfarin instructions into patient care documentation was available to physicians, but was misaligned to the standard warfarin instructions. In addition, the physician responsible for following up on a patient's first outpatient international normalized ratio was often omitted from the discharge instructions, potentially leaving patients without a physician to adjust their dosing appropriately. Adding this physician information, as well as aligning and updating all discharge instructions, allowed for clear, consistent patient instructions for home warfarin use. Moreover, EMR forms used by physicians and forms used by nurses to check for VTE prophylaxis were inconsistent, thus leading to potential confusion between physicians and nurses. Accordingly, regularly used EMR 


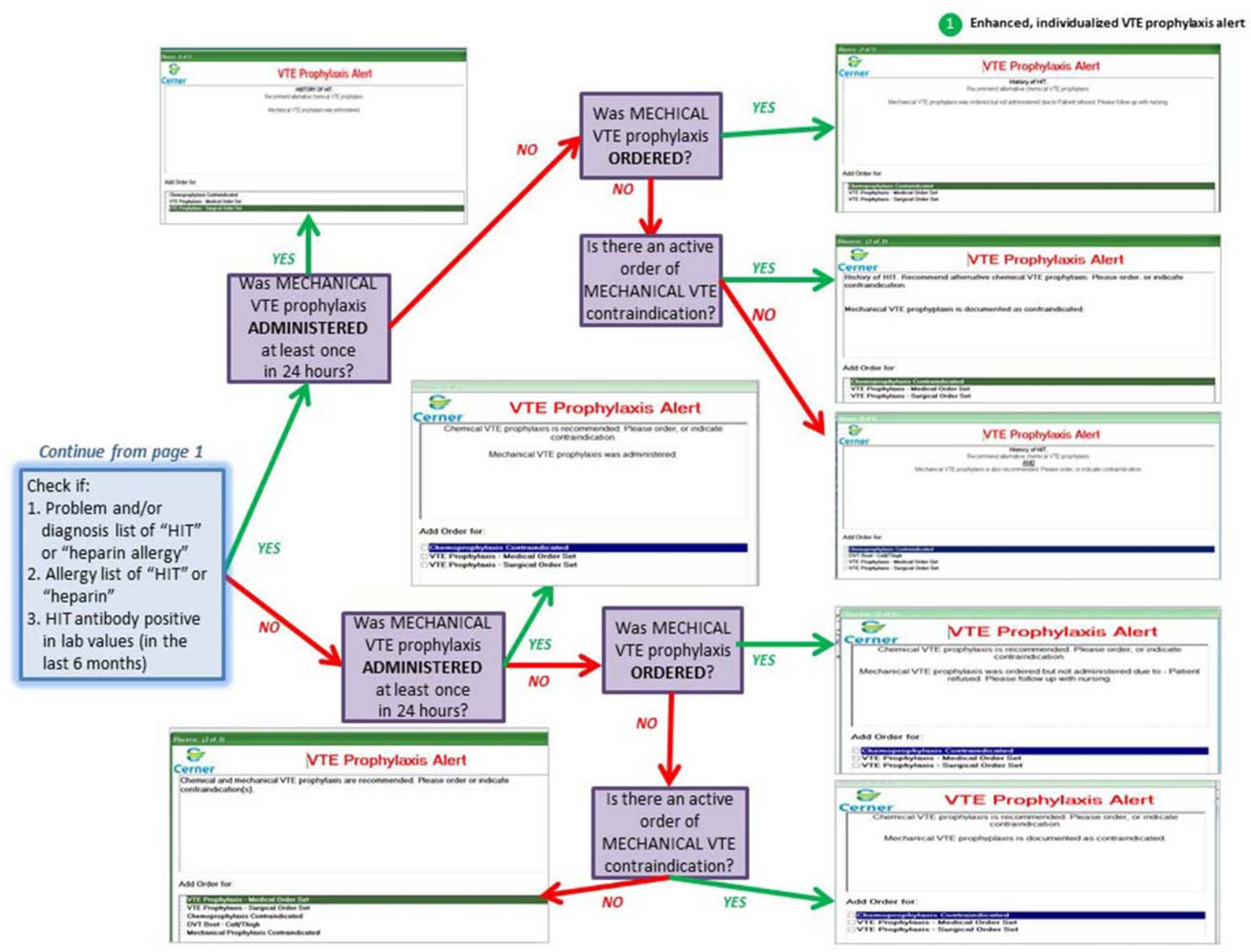

FIG. 1. Continued.

forms (eg, the interdisciplinary plan of care, and the unit summary page or unit snapshot) were updated and standardized.

\section{Control Mechanisms}

Concurrent with the implementation of the 15 interventions was the development of several control mechanisms to ensure sustained improvement. These mechanisms consisted of (1) an electronic proxy measure for VTE-1 (chemoprophylaxis) and (2) monitoring of clinician (including physicians, nurses, and midlevel providers) responses to the EMR alerts, and (3) a comprehensive EMR unit report (Figure 2).

Proxy Measure.Because the Joint Commission core measures are abstracted from only a sample of cases, and a time lag existed between each failure on VTE-1 (chemoprophylaxis) to the time the QI team learned of the failure, a proxy measure was created. This proxy measure is used as a stand-in for actual VTE-1 measure performance, but is generated in real time and reflects performance throughout the entire hospital instead of a random sample of cases. Using the Northwestern Electronic Data Warehouse (EDW), the
$\mathrm{NMH}$ analytics team created a report reflecting thromboprophylaxis administration on each hospital unit currently and over time. Performance could also be examined for each individual hospital service line. Being able to track longitudinal performance by unit and by service line enabled the QI team to understand trends in performance. Having the ability to examine patients who missed doses over the preceding few hours allowed unit leadership to proactively act upon the failures in a timely fashion, instead of waiting to receive their performance on the Joint Commission core measures.

Physician Alert Response Monitoring.Monitoring of clinical responses to EMR alerts was embedded as standard practice. Because alert fatigue is a documented unintended consequence of heavy reliance on EMR alerts, ${ }^{24,25}$ physicians and nurses who failed to respond to alerts regarding VTE prophylaxis were identified. Interventions targeted toward this group of nonresponders are currently being developed and tested.

EDW Unit Report. This report allows unit managers to track potential failures real time and act prior to a 


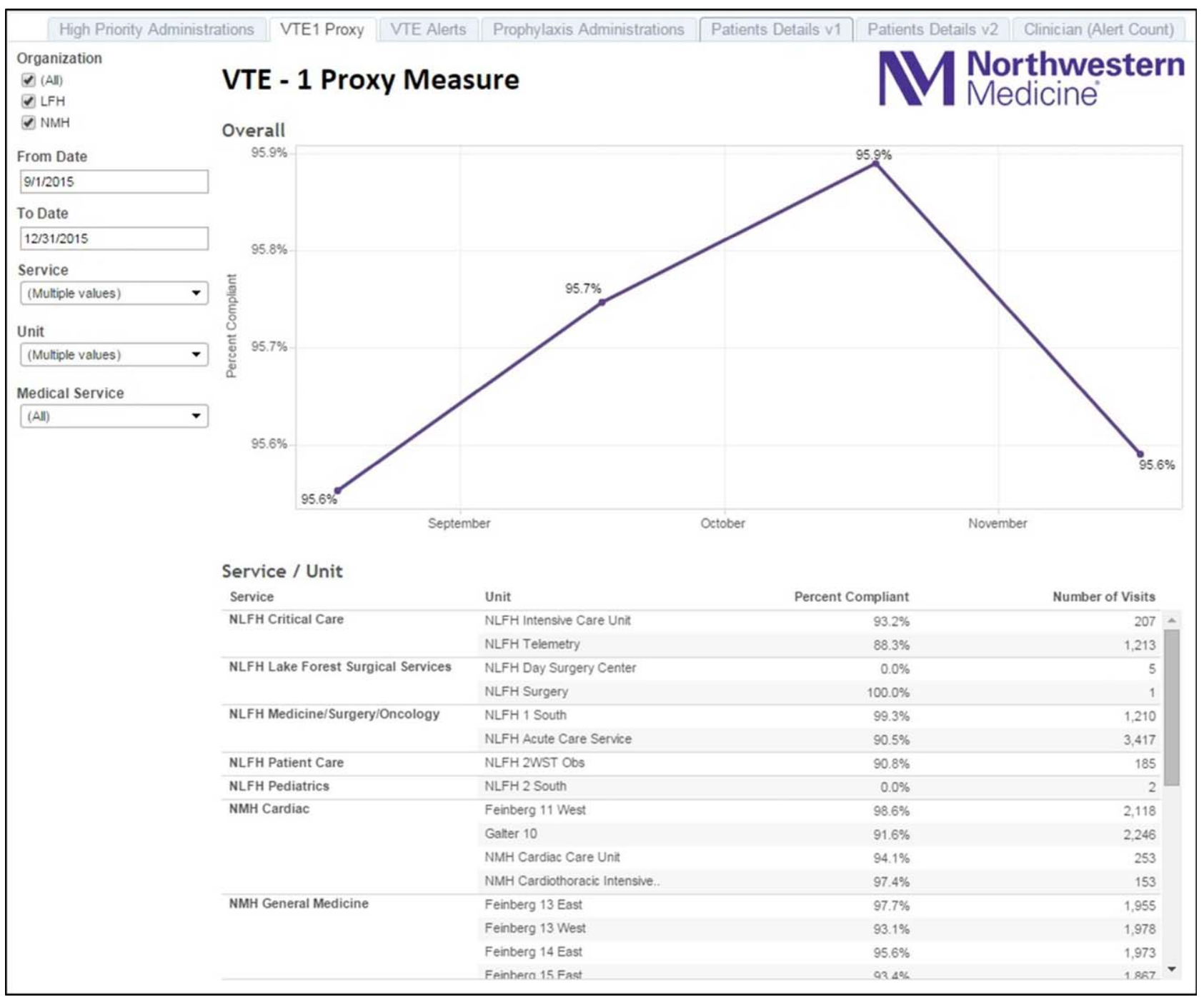

FIG. 2. VTE-1 proxy measure tab of the VTE report. Abbreviations: LFH, Lake Forest Hospital; NLFH, Northwestern Lake Forest Hospital; NMH, Northwestern Memorial Hospital; VTE, venous thromboembolism.

failure occurring (eg, missed chemoprophylaxis dose) through the NMH EDW (Figure 2). These reports contained detailed order and administration data at the individual patient, nurse, and physician levels. Missed doses of VTE chemoprophylaxis were immediately fed back to unit nursing managers who utilized the report to perform a rapid drilldown to identify the root cause(s) of the failure, and then rectify the failure while the patient was still hospitalized.

\section{Statistical Analyses}

Hospital performance on the VTE core measures and SCIP-VTE-2 was determined by trained nurse abstractors, who abstract cases randomly sampled by the University of HealthCare Consortium, and adjudicate findings as per the Specifications Manual for National Hospital Inpatient Quality Measures. Performance in the period prior to the QI intervention and in the period following the QI intervention was documented as proportions of abstracted cases found to be compliant with measure specifications. Differences between the pre- and postintervention periods were compared using a binomial test, with a $P$ value $<0.05$ considered significant. All analyses were performed using Stata version 13 (StataCorp, College Station, TX).

\section{RESULTS}

A total of 1679 cases were abstracted to obtain core measure performance in the time period before the DMAIC intervention phase (January 1, 2013-May 1, 2013), and 1424 cases were abstracted to obtain core measure performance in the time period after the DMAIC intervention phase (October 1, 2014-April 1, 2015).

Overall NMH performance on measures VTE-1 (chemoprophylaxis) and VTE3-6 (overlap therapy, platelet monitoring, warfarin discharge instructions, hospital-acquired [HA]-VTE) improved significantly $(P<0.05)$ (Table 1$)$. No improvement was seen on VTE-2 (intensive care unit chemoprophylaxis) given that pre- and postintervention performance was $100 \%$, which likely reflects previous hospital efforts 
to improve adherence to this measure. The percentage of patients who failed measure VTE-6 (number of patients with HA-VTE who did not have VTE prophylaxis ordered prior to diagnosis of their VTE) decreased from $8 \%$ to $2.4 \%$, demonstrating improved VTE prevention prescribing habits in $\mathrm{NMH}$ providers rather than a change in VTE event rates (ie, if more patients receive prophylaxis, they cannot be included in the numerator). Performance on SCIP-VTE-2 (perioperative chemoprophylaxis) increased from $99.5 \%$ to $100 \%$ as well but did not reach significance given the baseline high performance.

Measure performance on the general surgery services was comparable to the general medical services, with 1 exception. VTE-1 (chemoprophylaxis) performance was lower both prior to and following the QI intervention on general medicine services (medicine: $82.5 \%$ to $90.2 \%$ vs surgery: $94.4 \%$ to $97.6 \%$ ). Recent performance on the VTE-1 proxy measure has proven to be stable between $95 \%$ and $97 \%$ on surgery services. Physician response to alerts has increased slightly among the NMH general medicine practitioners $(15.2 \%-19.1 \%)$ but has been stable among NMH general surgery providers.

\section{DISCUSSION}

Our study demonstrates that a formal DMAIC QI project taken on by a multidisciplinary team (including clinicians from multiple specialties as well as personnel from IT, nursing, analytics, and PI) can be successfully implemented and can result in marked improvement in VTE core process measure performance. We used a multifaceted approach undertaken by the NMH VTE QI team, utilizing 15 data-driven interventions including EMR alerts, education initiatives, and new EMR order sets. These were combined with strong control mechanisms to sustain gains.

Previously published studies on VTE prophylaxis practices found that projects combining both passive (ie, helping clinicians to remember to risk-assess their patients' for VTE) and active (ie, assisting clinicians in appropriate prescribing practices) strategies are the most successful. ${ }^{26}$ Our improvement on VTE-1 can be compared to previous studies examining changes in ordering rates of VTE prophylaxis. Other QI projects that featured a combination of interventions observed similar significant increases in prophylaxis ordering. $^{27,28}$ Our improvement on VTE-1 (chemoprophylaxis) was significant, although the difference between pre- and postintervention performance varied by service type (general surgery vs general medicine vs other). The small increment of improvement on surgical services was likely attributable to a high baseline performance. Prior to 2013, surgically focused VTE prophylaxis QI efforts spurred by poor ACS-NSQIP performance proved to be successful, thus resulting in high surgical prophylaxis rates at the outset of the hospital-wide VTE DMAIC project.
One of the most significant unanticipated barriers to improving performance on VTE-1 (chemoprophylaxis) included the different hospital subcultures on the medical floors as compared to the surgical floors. The surgical floors had higher rates of compliance with VTE-1 than the general medicine floors both before and after the QI interventions. When the root causes were explored, the medical floors were found to have different ordering and administration patterns. These, in part, stemmed from differing guidelines ${ }^{29}$ and standards in the literature regarding VTE prophylaxis for medical and surgical patients. Multiple discussions within the multidisciplinary QI team and with each involved department were held, focusing on the data regarding safe care in medical patients at low risk for a VTE. Subsequent EMR alerts alterations reflected the internal medicine VTE prophylaxis recommendations for medical patients, allowing that low-risk patients could be assessed by the provider and given as a reason for foregoing VTE prophylaxis.

Barriers to VTE prophylaxis administration were encountered on the nursing front as well. Floor observations illustrated that chemoprophylaxis injections were often offered as an optional medication. Patients, when given the choice of receiving an injection or not, would understandably choose to forgo their heparin or enoxaparin shot. This missed dose was then documented as a patient refusal. This may not be a problem unique to $\mathrm{NMH}$; 1 study demonstrated that almost $12 \%$ of chemoprophylaxis doses may not be administered, and a frequent reason may be due to patient refusal. ${ }^{30}$ The lack of patient education regarding the importance of receiving chemical prophylaxis was an improvement opportunity at both the nursing and physician level. Not only did physicians and nurses take the responsibility to educate patients on the importance of receiving the proper prophylaxis, but nursing managers were made responsible for acting on missed doses that were listed on the real-time performance reports for their units. Missed prophylaxis doses thus became an actionable item instead of an acceptable occurrence.

Culture change in an organization is difficult and necessitates sustained efforts. An important component of our project is our control mechanism, in which a real-time, continuously updated unit report leverages data from our EDW to generate ongoing performance reports that are regularly reviewed by hospital leadership, clinical process owners, and, most importantly, frontline nurse managers. The unitspecific reports allow nurse managers and clinical project owners to review prophylaxis failures on a caseby-case basis daily and to address and rectify the cause. In addition, the QI team tracks individual physician action taken in response to EMR alerts. As performance feedback to surgical trainees has been demonstrated to have a positive effect on ordering practices, ${ }^{31}$ efforts to improve resident alert response 
rates by means of feedback and education are underway.

\section{Limitations}

Our results have to be interpreted within certain limitations. First, given that hospital performance on the VTE core measures is determined by abstracting only a sample of eligible cases, it is possible that our results were affected by sampling error. Second, because of problems with the VTE outcome measure due to surveillance bias, we are unable to draw any valid conclusions about changes in VTE event rates as a result of this QI project. Third, because many of our interventions were tailored to NMH's EMR platform and local hospital culture, it is possible that parts of our project are not readily generalizable to other hospitals; however, we believe that many components, such as the alert logics, can be easily tailored to other EMR platforms.

\section{CONCLUSION}

This institutional project was a large, multidisciplinary, and sustained undertaking that improved our performance on the VTE core measures. We believe that our bundle of EMR modifications, alerts (particularly the underlying alert logics), order sets, and standardization of summary EMR view can be adopted in other settings with appropriate adaptations to each hospital's specific local environment. Our focused educational interventions can also be easily adapted to other hospital settings. Perhaps the most important part of the project was the construction of novel control mechanisms that allow for tracking of physical alert response and for real-time evaluation, audit, and feedback of prophylaxis ordering and administration practices at NMH. Taken as a whole, this bundle of resources to improve adherence to optimal VTE prophylaxis will facilitate future interventions targeted at reaching defect-free care.

Disclosures: Nothing to report.

\section{References}

1. Guyatt GH, Akl EA, Crowther M, Gutterman DD, Schuunemann HJ. Executive summary: Antithrombotic Therapy and Prevention of Thrombosis, 9th ed: American College of Chest Physicians EvidenceBased Clinical Practice Guidelines. Chest. 2012;141:7S-47S.

2. Anderson FA Jr, Zayaruzny M, Heit JA, Fidan D, Cohen AT. Estimated annual numbers of US acute-care hospital patients at risk for venous thromboembolism. Am J Hematol. 2007;82:777-782.

3. Lefebvre P, Laliberte F, Nutescu EA, et al. All-cause and potentially disease-related health care costs associated with venous thromboembolism in commercial, Medicare, and Medicaid beneficiaries. J Manag Care Pharm. 2012;18:363-374.

4. Goldhaber SZ, Bounameaux H. Pulmonary embolism and deep vein thrombosis. Lancet. 2012;379:1835-1846.

5. Kahn SR, Solymoss S, Lamping DL, Abenhaim L. Long-term outcomes after deep vein thrombosis: postphlebitic syndrome and quality of life. J Gen Intern Med. 2000;15:425-429.

6. Prandoni P, Lensing AW, Cogo A, et al. The long-term clinical course of acute deep venous thrombosis. Ann Intern Med. 1996;125:1-7.
7. Streiff MB, Haut ER. The CMS ruling on venous thromboembolism after total knee or hip arthroplasty: weighing risks and benefits. JAMA. 2009;301:1063-1065.

8. Page RL II, Ghushchyan V, Gifford B, et al. Hidden costs associated with venous thromboembolism: impact of lost productivity on employers and employees. J Occup Environ Med. 2014;56(9):979-985.

9. Maynard G, Stein J. Designing and implementing effective venous thromboembolism prevention protocols: lessons from collaborative efforts. J Thromb Thrombolysis. 2010;29:159-166.

10. Collins R, Scrimgeour A, Yusuf S, Peto R. Reduction in fatal pulmonary embolism and venous thrombosis by perioperative administration of subcutaneous heparin. Overview of results of randomized trials in general, orthopedic, and urologic surgery. $N$ Engl J Med. 1988;318:1162-1173.

11. Dentali F, Douketis JD, Gianni M, Lim W, Crowther MA. Meta-analysis: anticoagulant prophylaxis to prevent symptomatic venous thromboembolism in hospitalized medical patients. Ann Intern Med. 2007;146:278-288.

12. Mismetti P, Laporte S, Darmon JY, Buchmuller A, Decousus H. Metaanalysis of low molecular weight heparin in the prevention of venous thromboembolism in general surgery. Br J Surg. 2001;88:913-930.

13. Haut ER, Lau BD, Kraus PS, et al. Preventability of hospital-acquired venous thromboembolism. JAMA Surg. 2015;150(9):912-915.

14. Kakkar AK, Cohen AT, Tapson VF, et al. Venous thromboembolism risk and prophylaxis in the acute care hospital setting (ENDORSE survey): findings in surgical patients. Ann Surg. 2010;251:330-338.

15. Deitelzweig SB, Lin J, Hussein M, Battleman D. Are surgical patients at risk of venous thromboembolism currently meeting the Surgical Care Improvement Project performance measure for appropriate and timely prophylaxis? J Thromb Thrombolysis. 2010;30:55-66.

16. Bilimoria KY, Chung J, Ju MH, et al. Evaluation of surveillance bias and the validity of the venous thromboembolism quality measure. JAMA. 2013;310:1482-1489.

17. Chung JW, Ju MH, Kinnier CV, Haut ER, Baker DW, Bilimoria KY. Evaluation of hospital factors associated with hospital postoperative venous thromboembolism imaging utilisation practices. BMJ Qual Saf. 2014;23(11):947-956.

18. Ju MH, Chung JW, Kinnier CV, et al. Association between hospital imaging use and venous thromboembolism events rates based on clinical data. Ann Surg. 2014;260:558-564; discussion 64-66.

19. Chung JW, Ju MH, Kinnier CV, Sohn MW, Bilimoria KY. Postoperative venous thromboembolism outcomes measure: analytic exploration of potential misclassification of hospital quality due to surveillance bias. Ann Surg. 2015;261(3):443-444.

20. Kinnier CV, Barnard C, Bilimoria KY. The need to revisit VTE quality measures. JAMA. 2014;312:286-287.

21. Bilimoria KY. Facilitating quality improvement: pushing the pendulum back toward process measures. JAMA. 2015;314:1333-1334.

22. Northwestern Medicine website. Available at: https://www.nm.org/ locations-at-northwestern-medicine. Accessed February 23, 2016.

23. Venous thromboembolism. The Joint Commission website. Available at: http://www.jointcommission.org/venous_thromboembolism. Accessed February 23, 2016.

24. Ash JS, Sittig DF, Campbell EM, Guappone KP, Dykstra RH. Some unintended consequences of clinical decision support systems. AMIA Annu Symp Proc. 2007:26-30.

25. van der Sijs H, Aarts J, Vulto A, Berg M. Overriding of drug safety alerts in computerized physician order entry. J Am Med Inform Assoc. 2006;13:138-147.

26. Tooher R, Middleton P, Pham C, et al. A systematic review of strategies to improve prophylaxis for venous thromboembolism in hospitals. Ann Surg. 2005;241:397-415.

27. Maynard GA, Morris TA, Jenkins IH, et al. Optimizing prevention of hospital-acquired venous thromboembolism (VTE): prospective validation of a VTE risk assessment model. J Hosp Med. 2010;5:10-18.

28. Gallagher M, Oliver K, Hurwitz M. Improving the use of venous thromboembolism prophylaxis in an Australian teaching hospital. Qual Saf Health Care. 2009;18:408-412.

29. Qaseem A, Chou R, Humphrey LL, Starkey M, Shekelle P. Venous thromboembolism prophylaxis in hospitalized patients: a clinical practice guideline from the American College of Physicians. Ann Intern Med. 2011;155:625-632.

30. Shermock KM, Lau BD, Haut ER, et al. Patterns of nonadministration of ordered doses of venous thromboembolism prophylaxis: implications for novel intervention strategies. PLoS One. 2013; 8:e66311.

31. Lau BD, Arnaoutakis GJ, Streiff MB, et al. Individualized performance feedback to surgical residents improves appropriate venous thromboembolism prophylaxis prescription and reduces potentially preventable VTE: a prospective cohort study [published online November 25, 2015]. Ann Surg. doi: 10.1097/SLA.0000000000001512. 